\title{
COMMENT ON MAGNETIC $\mathrm{e}^{+} \mathrm{e}^{-}$RESONANCES IN A CENTRAL FIELD
}

\author{
D.H. JAKUBASSA-AMUNDSEN ${ }^{1}$ \\ Physik-Department, Technische Universität München, 8046 Garching, FRG
}

Received 15 December 1986; revised manuscript received 21 January 1987; accepted for publication 21 January 1987

\begin{abstract}
Within a simple semiclassical model it is shown that the presence of an external Coulomb field induces only slight changes in the energy of a resonant electron-positron state unless for extremely small distances, while the width increases strongly with the field strength.
\end{abstract}

A great puzzle in present day physics is the interpretation of narrow lines in the spectrum of positrons emitted in very heavy ion collisions. Originally, these experimental investigations had been motivated by theory $[1,2]$ which predicts a spontaneous production of positrons as soon as the charge $Z$ of the combined nuclear system exceeds a critical value $\left(Z_{\mathrm{cr}}=173\right)$. The knowledge of both $Z$ and the time where the colliding nuclei are sufficiently close together, allows for an estimate of the structure of the resulting positron lines. The successful search for positron lines $[3,4]$ has been followed by a long series of experiments which investigated in detail the position and width of the lines, as well as their variation with system parameters [5]. It has, however, turned out that the presence of the lines for collision systems with $Z$ extending down to the subcritical region $(163 \leqslant Z \leqslant 188)$, as well as the tiny width of about 70 $\mathrm{keV}$, are in serious contradiction with the original picture of spontaneous positron production. More advanced theories which included nuclear resonance phenomena [6,7] or allowed for multiple electron excitation in a molecular-type picture [8] ran into difficulties as it became evident that the position of the lines did hardly change with $Z$. Thus, different production mechanisms have been suggested, among them the intermediate formation of a new particle $[9,10]$ or a three-lepton resonance $[11]$.

The recent observation of lines with the same

' Present address: Sektion Physik, Universität München, 8046 Garching, FRG. energy in both positron and electron spectra recorded in coincidence [12] stimulated an alternative explanation in terms of a decaying magnetic resonance of the $\mathrm{e}^{+} \mathrm{e}^{-}$binary system [13]. It was suggested [14] to describe the $\mathrm{e}^{+} \mathrm{e}^{-}$pair by the relativistic spinor equation [15]

$$
\begin{gathered}
\left\{c \alpha_{1} \cdot\left[p_{1}+(e / c) A_{1}\right]+c \alpha_{2} \cdot\left[p_{2}-(e / c) A_{2}\right]\right. \\
\left.+\beta_{1} m c^{2}+\beta_{2} m c^{2}+V_{0}-E\right\} \psi(\boldsymbol{r})=0,
\end{gathered}
$$

where indices 1 and 2 refer to the electron and positron, respectively, $V_{0}=-e^{2} / r$ is their mutual Coulomb interaction where $r=r_{1}-r_{2}$ is the relative coordinate of the pair, and $A_{1,2}=e \hbar\left(\sigma_{1,2} \times r\right) / 2 \mathrm{mcr}^{3}$ is the vector potential in a simple static approximation [14], $\sigma$ being the spin operator. It should be stressed that the Dirac-type equation (1) is a rather crude approximation to the quantum field theoretical many-body problem where the coupling between electron and positron proceeds in a highly nonlinear way through the radiation field. Effects like retardation or creation of additional pairs can thus not be described by (1) with the above choice of the interaction; it has, however, been shown in the specific case of an additional self-field that nonlinear coupling need not affect the existence of resonance states [16], and that some global effect of pair creation may be incorporated by means of a finite-range interaction between the two particles under consideration [15].

So despite of its deficiencies, eq. (1) may be used 
as a starting point for the investigation of magnetic resonances. The equation for the 16-component spinoir $\psi(\boldsymbol{r})$ can be expressed in terms of 4 coupled equations for the 4-component quantities $\psi_{\alpha}=$ $F_{1} \otimes F_{2}, \psi_{\beta}=F_{1} \otimes G_{2}, \psi_{;}=G_{1} \otimes F_{2}$ and $\psi_{\delta}=G_{1} \otimes G_{2}$, where $F$ and $G$ denote the large and small components of the one-particle Dirac spinor, respectively,

$\hat{D}_{1} \psi_{\eta}+\hat{D}_{2} \psi_{\beta}+\left(2 m c^{2}+V_{0}-E\right) \psi_{\alpha}=0$,

$\hat{D}_{1} \psi_{\beta}+\hat{D}_{2} \psi_{;}+\left(-2 m c^{2}+V_{0}-E\right) \psi_{\delta}=0$,

$\hat{D}_{1} \psi_{\delta}+\hat{D}_{2} \psi_{\alpha}+\left(V_{0}-E\right) \psi_{\beta}=0$,

$\hat{D}_{1} \psi_{\alpha}+\hat{D}_{2} \psi_{\delta}+\left(V_{0}-E\right) \psi_{\gamma}=0$,

where $\hat{D}_{1,2}=c \boldsymbol{p}_{1,2} \cdot \boldsymbol{\sigma}_{1,2}+e^{2} \hbar \boldsymbol{r} \cdot\left(\boldsymbol{\sigma}_{1} \times \boldsymbol{\sigma}_{2}\right) / 2 \mathrm{mcr}^{3}$.

Using the symmetry properties between electron and positron, a solution of (2) has been searched for by splitting eqs. (2c) and (2d) into four separate equations which only couple two functions each [14]. With the assumption that both particles have equal energy these equations are

$\hat{D}_{1} \psi_{\alpha}+\left[\frac{1}{2}\left(V_{0}-E\right)-m c^{2}\right] \psi_{\gamma}=0$,

$\hat{D}_{2} \psi_{\alpha}+\left[\frac{1}{2}\left(V_{0}-E\right)-m c^{2}\right] \psi_{\beta}=0$,

$\hat{D}_{2} \psi_{\delta}+\left[\frac{1}{2}\left(V_{0}-E\right)+m c^{2}\right] \psi_{\gamma}=0$,

$\hat{D}_{1} \psi_{\delta}+\left[\frac{1}{2}\left(V_{0}-E\right)+m c^{2}\right] \psi_{\beta}=0$.

The elimination of $\psi_{\gamma}$ and $\psi_{\beta}$ from (2a) with the help of ( $3 a$ ) yields an equation for $\psi_{\alpha}$ alone. In a similar way, ( $2 \mathrm{~b})$ leads to an equation for $\psi_{\delta}$ when ( $\left.3 \mathrm{~b}\right)$ is used. Both resulting equations are per construction symmetric with respect to the interchange of $\sigma_{1}$ and $\sigma_{2}$. Their solutions can be classified according to the total angular momentum $\boldsymbol{j}=\boldsymbol{l}+\boldsymbol{s}$ with $\boldsymbol{l}=\boldsymbol{r} \times \boldsymbol{p}_{1}$ $=-\boldsymbol{r} \times \boldsymbol{p}_{2}$ and $\boldsymbol{s}=\frac{1}{2}\left(\boldsymbol{\sigma}_{1}+\boldsymbol{\sigma}_{2}\right)$. Taking $j=0$ (the only case for which so far a resonance has been found), the angular part of $\psi_{\alpha}$ (and $\psi_{\delta}$ ) is given by the single state $|1 \mathrm{~s} j M\rangle=|1100\rangle$. With the ansatz $\psi_{\alpha, \delta}=f_{\alpha, \delta}|1100\rangle$ one is left with the radial equation

$$
\begin{aligned}
& {\left[\frac{V_{0}^{\prime}}{2\left[\frac{1}{2}\left(V_{0}-E\right) \mp m c^{2}\right]^{2}}\left(c^{2} \frac{\mathrm{d}}{\mathrm{d} r}+\frac{2 c^{2}}{r}-\frac{2-a}{6 m r^{2}}\right)\right.} \\
& -\frac{1}{\frac{1}{2}\left(V_{0}-E\right) \mp m c^{2}}\left(c^{2} \frac{\mathrm{d}^{2}}{\mathrm{~d} r^{2}}+\frac{2 c^{2}}{r} \frac{\mathrm{d}}{\mathrm{d} r}\right. \\
& \left.-\frac{2 c^{2}}{r^{2}}+\frac{4-a}{2 m r^{3}}-\frac{1}{2 m^{2} c^{2} r^{4}}\right) \\
& \left.-\left[\frac{1}{2}\left(V_{0}-E\right) \pm m c^{2}\right]\right] f_{\alpha, \delta}=0 .
\end{aligned}
$$

In this expression, the upper sign belongs to eq. (4a) for $f_{\alpha}$ and the lower sign to eq. (4b) for $f_{\delta}$. Further, $V_{0}^{\prime}=\mathrm{d} V_{0} / \mathrm{d} r$, and $a=-4$ is the angular eigenvalue of the tensor force $3\left(\sigma_{1} \cdot \hat{\boldsymbol{r}}\right)\left(\sigma_{2} \cdot \hat{\boldsymbol{r}}\right)-\sigma_{1} \cdot \sigma_{2}$ and simultaneously of the related operator $\left(\sigma_{1} \cdot \boldsymbol{r}\right)\left(\sigma_{2} \cdot \nabla\right)+$ $\left(\sigma_{2} \cdot \boldsymbol{r}\right)\left(\sigma_{1} \cdot \nabla\right)$ occurring later. The elimination of the first derivative of $f_{\alpha}$ leads to a Schrödinger-like equation with an effective, energy-dependent potential which supports one resonance state. The energy of that state was found by numerical quadrature to be $1.58 \mathrm{MeV}$ [14], compatible with twice the experimental positron peak energy.

In this letter the question is investigated whether in an external Coulomb field where the $\mathrm{e}^{+} \mathrm{e}^{-}$pair has to be created, the resonant state actually survives. Also, the consistency of eqs. (2) and (4) is commented on.

As one is interested in the creation of a resonance in heavy-ion collisions, the external field is chosen to be the two-center Coulomb field which depends on time through the internuclear coordinate $\boldsymbol{R}$. The lepton-nucleus interaction is taken of the form

$$
\begin{aligned}
V_{\mathrm{eN}}(\rho) & \sim 1 / \rho, \quad \rho>R_{\mathrm{K}}, \\
& \sim 1 / R_{\mathrm{K}}, \quad \rho \leqslant R_{\mathrm{K}},
\end{aligned}
$$

where $R_{\mathrm{K}}$ is the nuclear radius. In order to avoid the introduction of additional degrees of freedom into the equation for the electron-positron pair we shall restrict ourselves to spin-zero nuclei. Further, the monopole approximation to the two-center field is chosen. This is justified because pair creation requires strong fields which are only available at small $R$ where the monopole term of the potential dominates. With this approximation, the potential still depends on the relative orientation of the pair with respect to both $\boldsymbol{R}$ and $r_{0}$, the vector connecting the midpoints of the 


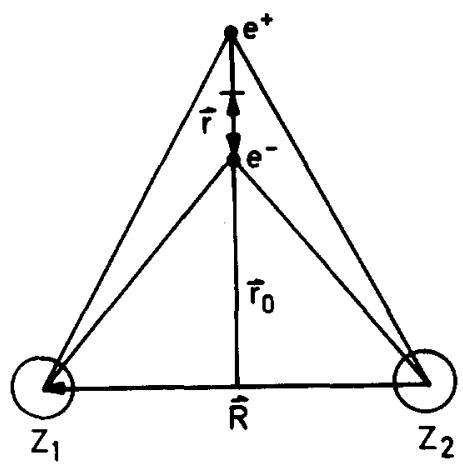

Fig. 1. Position of the electron-positron pair relative to the two nuclei (with charge $Z_{1}$ and $Z_{2}$, respectively) at equilibrium.

pair and the nuclei (cf. fig. 1). This orientation will be chosen in such a way that the potential acquires its minimum value, with $\boldsymbol{r}$ antiparallel to $r_{0}$ and perpendicular to $\boldsymbol{R}$. Moreover, the distance $r_{0}$ is determined by minimising the monopole field which in the region around the minimum is given by

$$
\begin{gathered}
V_{1}\left(r, r_{0}, R\right)=Z\left(\frac{1}{r_{0}+\frac{1}{2} r}-\frac{1}{R_{\mathrm{K}}}\right. \\
\left.+\frac{\left(\left|r_{0}-\frac{1}{2} r\right|+\frac{1}{2} R-R_{\mathrm{K}}\right)^{2}}{2\left|r_{0}-\frac{1}{2} r\right| R R_{\mathrm{K}}}\right) .
\end{gathered}
$$

Equal radii $R_{\mathrm{K}}$ of the two nuclei have been assumed. One is thus left with a potential which only depends on $R$ and $r$, while for each value of the two variables $r_{0}$ is computed numerically from $\mathrm{d} V_{1} / \mathrm{d} r_{0}=0$.

In order to investigate the behaviour of the electron-positron pair in this external field, we resort to the adiabatic approximation and calculate the wavefunction in the combined field $V=V_{0}+V_{1}$ at fixed $R$. After replacing $V_{0}$ in eq. (4a) by $V$ and eliminating the first derivative of $f_{\alpha}$ by means of the transformation $f_{\alpha}=\left(E+2 m c^{2}-V\right)^{1 / 2} \varphi_{\alpha} / r$ a Schrödinger-like equation for $\varphi_{\alpha}$ is obtained with an effective momentum $p(r, E)$ given by [14]

$$
\begin{aligned}
& c^{2} p^{2}(r, E) \equiv 2\left[E_{\mathrm{eff}}(E)-U_{\mathrm{eff}}(r, E)\right] \\
& =\left(\frac{V-E}{2}\right)^{2}-m^{2} c^{4}-\frac{2 c^{2}}{r^{2}} \\
& +\frac{4-a}{2 m r^{3}}-\frac{1}{2 m^{2} c^{2} r^{4}} \\
& -\frac{1}{E-V+2 m c^{2}}\left(\frac{c^{2} V^{\prime \prime}}{2}\right. \\
& \left.+\frac{\underline{3 c^{2} V^{\prime 2}}}{4\left(E-V+2 m c^{2}\right)}-\frac{c^{2} V^{\prime}}{r}+\frac{(2-a) V^{\prime}}{6 m r^{2}}\right),
\end{aligned}
$$

where the effective energy $E_{\mathrm{eff}}=\frac{1}{2}\left(\frac{1}{4} E^{2}-m^{2} c^{4}\right)$ and potential $U_{\text {eff }}$ have been introduced. In the WKB approximation, the energy $E$ of the resonant state is obtained from the quantisation of the action integral $\phi$, while its width $\Gamma$ is proportional to the barrier penetrability [17]

$\phi(E)=\int_{r_{\min }}^{r_{\max }} p \mathrm{~d} r=\frac{3}{2} \pi$,

$\Gamma=(2 \mathrm{~d} \phi / \mathrm{d} E)^{-1} \exp \left(-2 \int_{r_{\max }}^{r_{3}}|p| \mathrm{d} r\right)$.

In this expression, $r_{\min }, r_{\max }$ and $r_{3}$ are the three classical turning points. An interesting property of the effective momentum (10) is its weak dependence on energy in the region $r_{\min } \lesssim r \lessgtr r_{\max }$ where the resonant state is localised. This means that an increase of the effective energy implies a similar increase of the effective potential. As a consequence, the pair equation has to be solved very accurately for the wavefunction (going beyond WKB) in order to get a reliable estimate of the resonance energy. For this reason, the value of $a$ in eq. (4a) has been adjusted to -4.7 such that both energy and turning points from ref. [14] could be reproduced in the case of infinitely separated nuclei $(R \rightarrow \infty)$ where $E$ and $\Gamma$ attain the values of the isolated $\mathrm{e}^{+} \mathrm{e}^{-}$pair.

Fig. 2 shows energy and width of the resonance as a function of $R$ for the two systems $\mathrm{Pb}+\mathrm{Pb}(Z=164$, $\left.R_{\mathrm{K}}=7 \mathrm{fm}\right)$ and $\mathrm{U}+\mathrm{U}\left(Z=184, R_{\mathrm{K}}=7.32 \mathrm{fm}\right)$. When the nuclei approach each other, $E$ increases slightly, and eventually drops very fast when they come into 


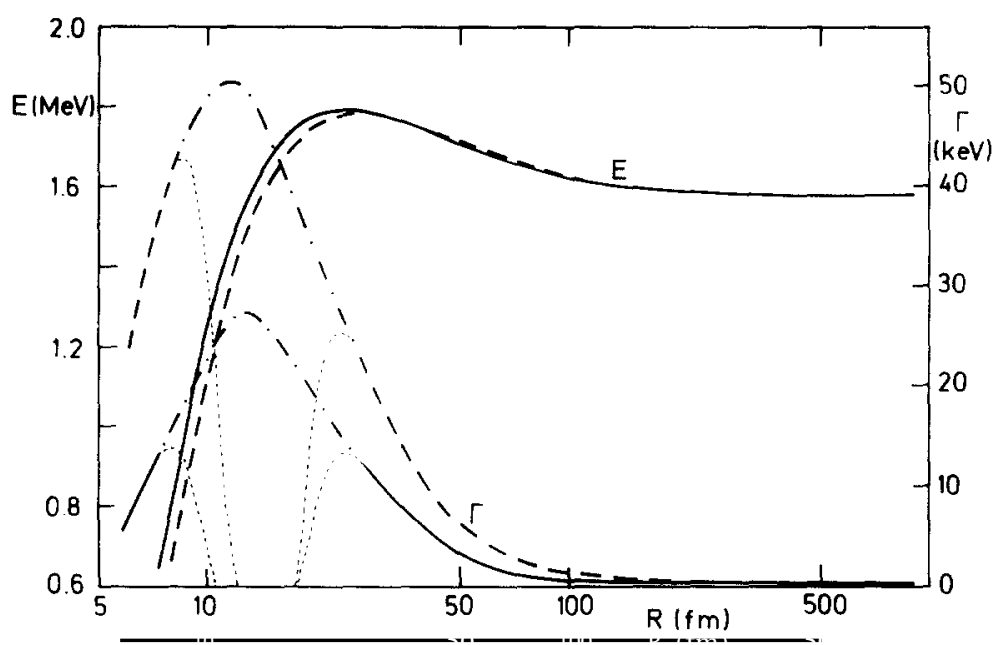

Fig. 2. Energy $E$ (left scale) and width $\Gamma$ (right scale) of the $\mathrm{e}^{+} \mathrm{e}^{--}$state as a function of the internuclear distance $R$. Full lines, $Z=164$, dashed lines, $Z=184$. For more details, see text.

contact and exert a strong attractive force on the pair. The energy depends not only weakly on $R$ (for $R>2 R_{\mathrm{K}}$ ) but also weakly on the combined nuclear charge. The reason for this behaviour is the localisation of the resonant state at extremely small distances $\left(r_{\max } \sim 5 \mathrm{fm}\right)$. There, the magnetic force between electron and positron exceeds by far the external field such that the influence of the latter is weak as long as the two nuclei do not penetrate each other.

The situation is quite different as far as the width of the resonance is concerned. Through its dependence on the outer turning point which lies at rather large distances (decreasing with $R$ from 400 to 40 fm) $\Gamma$ shows strong variations with $R$ as well as with $Z$. Moreover, while the energy depends only slightly on the particular choice of the potential inside the nucleus (as long as it is finite), $\Gamma$ is very sensitive already at $R$ considerably larger than $2 R_{\mathrm{K}}$. In particular, the vanishing width around $R=2 R_{\mathrm{K}}$ (dotted line in fig. 2) is an artifact due to the (spurious) divergence of the second derivative of the potential at $R_{\mathrm{K}}$ (cf. eq. (8)) which enters into the effective momentum (10) in the region of the outer turning point. Rather, the width will follow the dash-dotted line if a smooth potential is chosen. However, a precise knowledge of the potential inside the nucleus is required to determine the width for small $R$ sufficiently accurately.
Having established the survival of the resonant state in an external Coulomb field, the next point is its production in heavy-ion collisions. it will take place via (at least) a second-order process because the pair wavefunction vanishes at $r=0$. Taken into consideration that $Z / 137$ exceeds unity this means no severe reduction of the production probability. In terms of the adiabatic monopole approximation used above, one of the couplings to the vacuum will proceed via the time-variation of the monopole Coulomb field (which is included in the wave function), while the other coupling is induced by the potential not included in the wavefunction (the dipole part in our case of an $l=1$ resonance). It should be noted that the cross section for positron emission which eventually has to be calculated in order to compare with experiment, depends significantly on the decay probability of the resonance, i.e. on its width [18].

However, such calculations are not meaningful at this stage. The reason lies in the fact that the magnetic resonance discussed above lacks its mathematical foundation as the respective wavefunction cannot be derived from the original equations (2).

In order to prove this, it is assumed that the solution of $(4)$ is also a solution of (2). If this were true, the functions $\psi_{\alpha, \delta}=f_{\alpha, \delta}|1100\rangle$ with $f_{\alpha, \delta}$ from (4) should obey the additional conditions which are obtained from eqs. (3a) and (3b) by eliminating $\psi_{\gamma}$ and $\psi_{\beta}$. These conditions can be expressed in terms 
of two coupled equations for $\psi_{\alpha}$ and $\psi_{\delta}$ which are symmetric in $\sigma_{1}$ and $\sigma_{2}$. It is straightforward to show that also the solutions to these equations can be classified according to $j$. Thus, the angular part of $\psi_{\alpha}$ and $\psi_{\delta}$ is correctly given by the state $|1100\rangle$. If this particular state is inserted, one of the coupled equations becomes a trivial identity, while the other one leads to a supplementary condition on $f_{\alpha}$ and $f_{\delta}$ :

$$
\begin{aligned}
& \frac{1}{\frac{1}{2}\left(V_{0}-E\right)-m c^{2}}\left(r \frac{\mathrm{d}}{\mathrm{d} r}+2-\frac{1}{m c^{2} r}\right) f_{\alpha} \\
& =\frac{1}{\frac{1}{2}\left(V_{0}-E\right)+m c^{2}}\left(r \frac{\mathrm{d}}{\mathrm{d} r}+2-\frac{1}{m c^{2} r}\right) f_{\delta} .
\end{aligned}
$$

Upon operating with $\mathrm{d} / \mathrm{d} r$ on (5) and eliminating all derivatives with the help of $(4 a),(4 b)$ and $(5)$, the relation between $f_{\alpha}$ and $f_{\delta}$ turns into

$$
\left[\frac{1}{2}\left(V_{0}-E\right)-m c^{2}\right] f_{\delta}=\left[\frac{1}{2}\left(V_{0}-E\right)+m c^{2}\right] f_{\alpha} \text {. }
$$

This relation can be used to replace $f_{\delta}$ in $(4 \mathrm{~b})$ by $f_{\alpha}$ which leads to the following equation,

$$
\begin{aligned}
& \left\{\frac { \underline { V _ { 0 } ^ { \prime } } } { 2 V _ { + } V _ { - } } \left[\left(1+\frac{4 m c^{2}}{V_{-}}\right) c^{2} \frac{\mathrm{d}}{\mathrm{d} r}\right.\right. \\
& \left.\quad+\frac{2 c^{2}}{r}\left(1+\frac{2 m c^{2}}{V_{-}}\right)-\frac{2-a}{6 m r^{2}}\right] \\
& +\frac{m c^{4}}{V_{+} V_{-}^{2}}\left[V_{0}^{\prime \prime}-V_{0}^{\prime 2}\left(\frac{1}{2 V_{+}}+\frac{1}{V_{-}}\right)\right] \\
& \quad-\frac{1}{V_{-}}\left(c^{2} \frac{\mathrm{d}^{2}}{\mathrm{~d} r^{2}}+\frac{2 c^{2}}{r} \frac{\mathrm{d}}{\mathrm{d} r}-\frac{2 c^{2}}{r^{2}}\right. \\
& \left.\left.+\frac{4-a}{2 m r^{3}}-\frac{1}{2 m^{2} c^{2} r^{4}}\right)-V_{+}\right\} f_{\alpha}=0,
\end{aligned}
$$

with $V_{ \pm}=\frac{1}{2}\left(V_{0}-E\right) \pm m c^{2}$. This equation is similar but not identical to (4a). The terms containing the second derivative of $f_{\alpha}$ coincide in (4a) and (7), whereas the terms proportional to $\mathrm{d} f_{\alpha} / \mathrm{d} r$ do not, and there are no common solutions to (4a) and (7). The consequence of this is that the radial function $f_{\alpha}$ from (4a) is not solution to the original equations (1) or (2).

Although new calculations which avoid any manipulation on the structure of (2) seem to indi- cate that the resulting potential does not support a resonant state for $j=0$ [19], the possibility of finding magnetic resonances is of course still open. For such investigations, it would be advisable to use more elaborate equations than (2) as a starting point.

With this situation, the above discussion of the resonance from eq. (4) reduces to a case study. However, as all magnetic resonances tend to be confined to spatial extensions of nuclear, rather than atomic size [13,14], the implications of the model case will have a general validity. This concerns the fact that the energy of the resonant state is strongly correlated with its value in the field-free case, whereas the width increases by more than one order of magnitude when the Coulomb field is switched on (with an estimated peak value around $50 \mathrm{keV}$ ). Thus, if a true magnetic resonance should show up at an energy around 1.6 $\mathrm{MeV}$, so that it could be considered as explanation of the experimental positron peak at $\sim 300 \mathrm{keV}$, the width would not be inconsistent with the experimental observation provided that in the field-free case, $\Gamma$ were well below this value.

I would like to thank P.A. Amundsen, B. Müller and $P$. Kienle for enlightening discussions.

\section{References}

[1] Ya.B. Zeldovich and V.S. Popov, Sov. Phys. Usp. 14 (1972) 673.

[2] B. Müller, J. Rafelski and W. Greiner, Z. Phys. 257 (1972) $62,183$.

[3] J. Schweppe, A. Gruppe, K. Bethge, H. Bokemeyer, T. Cowan, H. Folger, J.S. Greenberg, H. Grein, S. Ito, R. Schule, D. Schwalm, K.E. Stiebing, N. Trautmann, P. Vincent and M. Waldschmidt, Phys. Rev. Lett. 51 (1983) 2261.

[4] M. Clemente, E. Berdermann, P. Kienle, H. Tsertos, W. Wagner, C. Kozhuharov, F. Bosch and W. Koenig, Phys. Lett. B 137 (1984) 41.

[5] P. Kienle, Annu. Rev. Nucl. Part. Sci. 36 (1986).

[6] U. Heinz, U. Müller, J. Reinhardt, B. Müller and W. Greiner, Ann. Phys. 158 (1984) 476

[7] T. Tomoda and H.A. Weidenmüller, Phys. Rev. C 28 (1983) 739.

[8] W. Lichten and A. Robatino, Phys. Rev. Lett. 54 (1985) 781.

[9] A. Schäfer, J. Reinhardt, B. Müller, W. Greiner and G. Soff, J. Phys. G 11 (1985) L69.

[10] A.B. Balantekin, C. Bottcher, M.R. Strayer and S.J. Lee, Phys. Rev. Lett. 55 (1985) 461. 
[11] C.Y. Wong, Phys. Rev. Lett. 56 (1986) 1047.

[12] T. Cowan, H. Backe, K. Bethge, H. Bokemeyer, H. Folger, J.S. Greenberg, K. Sakaguchi, D. Schwalm, J. Schweppe, K.E. Stiebing and P. Vincent, Phys. Rev. Lett. 56 (1986) 444.

[13] A.O. Barut and J. Kraus, Phys. Lett. B 59 (1975) 175.

[14] C.Y. Wong and R.L. Becker, ORNL preprint (1986).
[15] E. Fermi and C.N. Yang, Phys. Rev. 76 (1949) 1739.

[16] A.O. Barut and J. Kraus, Phys. Rev. D 16 (1977) 161.

[17] M.R.C. McDowell and J.P. Coleman, Introduction to the theory of ion-atom collisions (North-Holland, Amsterdam, 1970) p. 81 .

[18] D.H. Jakubassa and M. Kleber, Z. Phys. A 277 (1976) 41. [19] X. Geiger and B. Müller, private communication (1986). 